OPEN ACCESS

Check for updates

For numbered affiliations see end of the article.

Correspondence to: O A Olavarria oscarolavarria@hotmail.com (or @oolavarriak on Twitter: ORCID 0000-0001-87315395)

Additional material is published online only. To view please visit the journal online.

Cite this as: $B M / 2020 ; 370: m 2457$ http://dx.doi.org/10.1136 bmj.m2457 Accepted: 4 May 2020

\section{Robotic versus laparoscopic ventral hernia repair: multicenter, blinded randomized controlled trial}

\author{
Oscar A Olavarria, ${ }^{1,2}$ Karla Bernardi, ${ }^{1,2}$ Shinil K Shah,, ${ }^{1,3}$ Todd D Wilson, ${ }^{1,3}$ Shuyan Wei, ${ }^{1,2}$ \\ Claudia Pedroza, ${ }^{4,5}$ Elenir B Avritscher, ${ }^{4,5}$ Michele M Loor, ${ }^{6}$ Tien C Ko, ${ }^{1}$ Lillian S Kao, ${ }^{1,2}$ \\ Mike K Liang ${ }^{1,2}$
}

\section{ABSTRACT}

OBJECTIVE

To determine whether robotic ventral hernia repair is associated with fewer days in the hospital 90 days after surgery compared with laparoscopic repair. DESIGN

Pragmatic, blinded randomized controlled trial.

SETTING

Multidisciplinary hernia clinics in Houston, USA.

PARTICIPANTS

124 patients, deemed appropriate candidates for elective minimally invasive ventral hernia repair, consecutively presenting from April 2018 to February 2019.

INTERVENTIONS

Robotic ventral hernia repair $(n=65)$ versus

laparoscopic ventral hernia repair $(n=59)$.

MAIN OUTCOME MEASURES

The primary outcome was number of days in hospital within 90 days after surgery. Secondary outcomes included emergency department visits, operating room time, wound complications, hernia recurrence, reoperation, abdominal wall quality of life, and costs from the healthcare system perspective. Outcomes were pre-specified before data collection began and analyzed as intention to treat.

RESULTS

Patients from both groups were similar at baseline. Ninety day follow-up was completed in 123 (99\%) patients. No evidence was seen of a difference in days in hospital between the two groups (median 0 $\checkmark 0$ days; relative rate $0.90,95 \%$ confidence interval 0.37 to $2.19 ; \mathrm{P}=0.82$ ). For secondary outcomes, no differences were noted in emergency department visits, wound complications, hernia recurrence, or

\section{WHAT IS ALREADY KNOWN ON THIS TOPIC}

A retrospective national database study comparing robotic versus laparoscopic ventral hernia repair showed a shorter postoperative hospital length of stay with robotic repair

No differences in clinical outcomes were reported

However, no randomized controlled trials have been conducted to corroborate these findings

\section{WHAT THIS STUDY ADDS}

Compared with laparoscopic ventral hernia repair, robotic repair did not decrease length of stay, nearly doubled operating room time, and significantly increased cost of healthcare

This was the case even without accounting for acquisition and maintenance costs of the robot reoperation. However, robotic repair had longer operative duration (141 $v 77 \mathrm{~min}$; mean difference $62.89,45.75$ to $80.01 ; P \leq 0.001)$ and increased healthcare costs ( $\$ 15865$ ( $£ 12746 ; € 14125) v$ $\$ 12955$; cost ratio $1.21,1.07$ to 1.38 ; adjusted absolute cost difference $\$ 2767, \$ 910$ to $\$ 4626$; $\mathrm{P}=0.004)$. Among patients with robotic ventral hernia repair, two had an enterotomy compared none with laparoscopic repair. The median one month postoperative improvement in abdominal wall quality of life was 3 with robotic ventral hernia repair compared with 15 following laparoscopic repair.

\section{CONCLUSION}

This study found no evidence of a difference in 90 day postoperative hospital days between robotic and laparoscopic ventral hernia repair. However, robotic repair increased operative duration and healthcare costs.

TRIAL REGISTRATION

Clinicaltrials.gov NCT03490266.

\section{Introduction}

In the past decade, the use of robotic platforms in surgery has grown exponentially, reaching a fourfold increase in the number of procedures performed worldwide with robotic assistance from 200000 in 2009 to nearly 900000 procedures a year in $2017 .{ }^{1}$ General surgery has seen the fastest growth of all surgical specialties, and hernia repairs are among the most commonly performed general surgery procedures. This growth occurred despite limited evidence supporting the practice of robotic ventral hernia repair (RVHR). To date, only studies with designs at high risk for bias have been published: no randomized controlled trials assessing RVHR have been published. ${ }^{2}$

Recently, a retrospective national database study published by the Americas Hernia Society Quality Collaborative compared the most commonly performed minimally invasive ventral hernia repair techniques: RVHR with intraperitoneal onlay mesh placement and laparoscopic ventral hernia repair (LVHR) with intraperitoneal onlay mesh placement. This study showed a shorter postoperative hospital length of stay with RVHR (0 days in the RVHR group versus 1 day in the LVHR group; $\mathrm{P} \leq 0.001$ ) and no differences in safety or clinical outcomes. ${ }^{3}$ Our aim was to validate these findings by doing a randomized controlled trial to compare the clinical and patient centered outcomes between RVHR and LVHR. We hypothesized that RVHR as opposed to LVHR would decrease days in the hospital up to 90 days after surgery. 


\section{Methods}

We did a multicenter, multi-blinded, randomized controlled trial after obtaining institutional review board approval. Participating centers included Memorial Hermann Hospital System and Lyndon Baines Johnson General Hospital in Houston, Texas. We followed the Consolidated Standards of Reporting Trials (CONSORT) guidelines, and the study was posted in clinicaltrials. gov (NCT03490266).

\section{Eligibility}

We approached patients consecutively presenting to multidisciplinary hernia clinics from April 2018 to February 2019 who were deemed appropriate candidates for elective minimally invasive ventral hernia repair. We have previously published definitions for these criteria. ${ }^{4}$ Patients who voluntarily agreed to participate in the study signed a surgical informed consent and a separate research consent before enrollment. We included adult patients ( $>18$ years old) with a ventral hernia defect less than $12 \mathrm{~cm}$ wide on physical examination, who would likely tolerate pneumoperitoneum (for example, no advanced chronic obstructive pulmonary disease or congestive heart failure), with no history of open abdomen or extensive lysis of adhesions for bowel obstruction, and with no active infection such as mesh infection.

We excluded patients with severe comorbid conditions that would likely limit survival beyond two years as judged by a surgeon (for example, advanced cirrhosis or metastatic cancer), patients unlikely to follow up (for example, lives out of state or no phone), and non-English and non-Spanish speakers. We defined the inclusion and exclusion criteria during our study design phase, before the study started, and made no changes after enrollment started.

\section{Data collection and follow-up}

We obtained baseline demographic information from patients who agreed to participate in the study through a full medical history and standardized physical examination during preoperative clinic appointments. Before the start of this randomized controlled trial, we did qualitative research directed at identifying and understanding what factors patients consider to be important during decision making related to the management of their ventral hernias. ${ }^{5}{ }^{6}$ Among the factors outlined by patients, quality of life and pain were considered the most important variables within their expectations. During our study, patient centered outcomes including abdominal wall quality of life and pain scores were obtained preoperatively and one month postoperatively. Patients were asked to complete the modified Activity Assessment Scale, a validated, hernia specific questionnaire that investigates patients' abdominal wall quality of life through a 12 question survey. ${ }^{78}$ These questions explore different domains of quality of life including physical activity and social and psychological wellbeing. Survey responses are normalized to a scale ranging from 1 (poor) to 100 (perfect), with normal abdominal wall quality of life being 80 or greater. The minimal clinically important difference is 7 for a minor change and 14 for a major change. ${ }^{910}$ Preoperative pain scores were assessed with a visual analog scale ranging from 0 (no pain) to 10 (worst possible pain); the minimal clinically important difference is $1 .^{11}$

\section{Intervention}

Only surgeons experienced in minimally invasive hernia were allowed to participate in the study. ${ }^{12}$ The three participating surgeons did both RVHR and LVHR for patients included in this study. Senior residents (postgraduate year 4 and 5) with variable experience in robotic surgery were allowed to assist in the operation at the discretion of the surgeon. Despite their previous experience, our surgeons and teams completed a "ramp-up" period of at least 50 standardized cases per site before the start of this study. The literature has reported wide ranges of suggested numbers of cases to achieve a plateau in performance (for example, 25-75 colorectal cases, $10-95$ bariatric cases, and $10-80$ solid organ cases), but no consensus exists on the number of hernia repairs a surgeon should do to be proficient or to be an expert. ${ }^{1314}$ We selected 50 cases for our ramp-up period to ensure that operating room teams and surgeons had optimized workflow and to mitigate any risk of longer operative time and complications associated with a possible "learning curve" effect of the standardized ventral hernia repair technique used for this study. Patients in the "ramp-up" period were not included in this study; however, we reviewed and published our previous clinical experience with robotics to establish a baseline of expertise. ${ }^{1516}$

Ventral hernia repairs were performed using conventional approaches recommended by surgical societies. ${ }^{17} 18$ Common steps of both procedures were as follows: the abdomen was entered in the right or left upper abdominal quadrant with an optical trocar; a pneumoperitoneum was created by insufflating the abdomen with $\mathrm{CO}_{2}$ to an intra-abdominal pressure of $10 \sim 15 \mathrm{~mm} \mathrm{Hg}$; two additional trocars were placed under direct visualization; all anterior abdominal wall adhesions were dissected free; mesh was inserted through a $12 \mathrm{~mm}$ port; the hernia defect was closed with 0-polydioxanone; a mid-density hydrogel adhesion barrier coated polypropylene mesh was used; mesh size was selected to provide at least $5 \mathrm{~cm}$ of overlap on all sides relative to the hernia defect and was then secured to the anterior abdominal wall through an intraperitoneal onlay mesh placement technique; and the skin was closed with absorbable sutures and dressed with cyanoacrylate glue (supplementary table A). RVHR was performed using three 8-12 mm ports placed along the lateral abdomen, the robotic platform was docked, all adhesions were taken down with robotic scissors and grasper, the fascial defect was closed with 0 locking barbed polydioxanone suture, the mesh was secured intraperitoneally with circumferential running 2-0 barbed polydioxanone suture, and all $12 \mathrm{~mm}$ ports were closed with 0-polyglactin 910 suture. LVHR was performed using 
three 5-12 mm ports, all adhesions were taken down with laparoscopic scissors or a vessel sealing device, the fascial defect was closed with 0-polydioxanone sutures, and mesh was secured intraperitoneally with trans-fascial sutures and a circumferential double crown of permanent tacks. We did not consider bridging the hernia defect, as primary fascial closure has been associated with greater improvement in quality of life and lower seroma rates with no significant difference in pain scores. ${ }^{19-21}$ Peri-operative multi-modal pain management and enhanced recovery practices were used and standardized (supplementary table B). ${ }^{4}$

Randomization, blinding, and outcome assessment An independent research assistant randomized patients in a 1:1 allocation ratio to either RVHR or LVHR by using a computer generated variable block randomization schema stratified by surgeon. On the day of surgery, surgeons contacted the research assistant, who determined the treatment allocation through opening of sequentially numbered opaque sealed envelopes. Owing to the nature of the intervention, operating surgeons and the research coordinator who determined the randomization allocation could not be blinded. However, the patient and the rest of the research team, including postoperative outcome assessors, were all blinded to the patients' allocation group.

All patients were scheduled for follow-up at the clinic one month after their procedure. Future long term follow-up is planned. Surgeons blinded to the treatment arms, different from the operating surgeon, did a standardized physical examination with emphasis on the identification of complications including surgical site infections, surgical site occurrences (for example, seromas, hematomas, wound dehiscence), and hernia recurrence. Additional radiographic imaging was obtained on demand; if signs or symptoms (for example, pain, bulging, or palpable abdominal wall mass) on the history and physical examination concerning for possible complications occurred, the outcome assessor ordered a computed tomography scan of the abdomen and pelvis. During each evaluation, patients were also asked to complete the modified Activity Assessment Scale survey and visual analog scale.

\section{Outcomes}

We defined the primary outcome as number of days in hospital (overnight stay) at 90 days after surgery, including postoperative and readmission length of stay. Secondary outcomes included: operating room duration defined as incision to skin closure time, surgical site infections, surgical site occurrences, hernia recurrence, reoperation, Clavien-Dindo complication grades, emergency department visits, change in abdominal wall quality of life, change in visual analog scale pain scores, and costs from the healthcare system perspective. One specific Clavien-Dindo complication, enterotomy, unexpectedly seemed to show possible differences. For this reason, we reported enterotomy in the results despite it not being pre-specified. We defined surgical site infections, per the Centers for Disease Control and Prevention guidelines, as being either incisional or organ/space infections; we further subdivided incisional surgical site infections into superficial if involving only the skin and subcutaneous tissue and deep if involving deeper soft tissue, and we defined organ/space infections as those involving any part other than the incised abdominal wall layers. ${ }^{22}$ Surgical site occurrences included seromas, hematomas, and wound dehiscences. We defined a seroma as a mass or swelling in the wound caused by the localized accumulation of fluid without signs of surgical site infection, a hematoma as a solid swelling of clotted blood within the tissues, and a dehiscence as a disruption of the surgical wound of any size. We defined reoperation as an unanticipated return to the operating room for complications related to the index operation. ${ }^{23}{ }^{24}$ We assessed surgical site infections, surgical site occurrences, and recurrence at the one month postoperative clinic visit, whereas readmissions, emergency department visits, reoperations, and other complications were assessed through review of electronic medical records up to 90 days after the index surgery. Costs including all patient visits, admissions, and procedural costs from the operation through the first 90 postoperative days came from the hospital administration accounting system. Cost did not include surgeons' fees or the initial acquisition cost of the robotic or laparoscopic platforms.

\section{Analysis}

Three authors (OAO, CP, and EBA) who were not involved in recruitment or data collection did intention to treat analyses for all outcomes. Frequentist analysis of the primary outcome used a negative binomial generalized linear model with the dependent variable of hospital length of stay adjusted for the randomization variable (RVHR $v$ LVHR) and stratification variable (surgeon). Secondary outcomes were analyzed using generalized linear models for continuous outcomes and logistic modified Poisson regressions for binary outcomes. Costs were analyzed through generalized linear models with $\log$ link and $\gamma$ distribution. ${ }^{25}$ All models included the randomization and stratification variables as covariates. $^{26} 27$ The regression mean differences (coefficients) of these models were exponentiated to estimate the relative rates and 95\% confidence intervals. We used analysis of covariance to analyze abdominal wall quality of life and pain scores. ${ }^{28}$ We did subgroup analyses of operative duration and costs, planned a priori, including main effects for the intervention group, subgroup variables one at a time (hernia type, hernia area, and surgeon), and their interaction. We did multivariable generalized linear model regression analyses for these outcomes using the same subgroup variables (hernia type, hernia area, and surgeon) to allow risk adjusted interpretation of the results.

No conventionally agreed minimum clinically important difference exists for hospital length of stay 
after ventral hernia repair; however, considering that most of these procedures are done on an outpatient basis, we believe that a difference of one day between groups would be an important difference, as hospital stay affects costs, reimbursement, and healthcare use. Furthermore, a previous study from the Americas Hernia Society database showed that RVHR was associated with shorter length of stay in hospital by one day ( 0 days for RVHR versus 1 day for LVHR; $\mathrm{P} \leq 0.001){ }^{3}$ Therefore, we selected a one day difference for our sample size calculation. Assuming a difference in the primary outcome of zero versus one day with a dispersion of three days, a $10 \%$ dropout rate, a two sided $\alpha$ of 0.05 , and $80 \%$ power, we needed at least 120 patients to be randomized. All patients who were enrolled and consented were randomized. Thus, four additional patients who were already consented proceeded with randomization despite our sample size having been achieved. This was approved by the institutional review board. We used the computing environment $R$ version 3.6.0 for all statistical analyses, along with the following extension packages: rstanarm, brms, lme4, and geepack. ${ }^{29}$

\section{Patient and public involvement}

This research was supported by patient focus groups that provided input to the program of research. Patients partnered with us for the design of the study, including the burden of the intervention from the patient's perspective as well as outcomes important to the patient. However, patients were not invited to contribute to the data collection or analysis or the writing or editing of this document for readability or accuracy.

\section{Results}

We approached 175 eligible patients, of whom 124 were randomized; 65 underwent RVHR and 59 LVHR (fig 1). Most patients were women and of Hispanic or African-American race/ethnicity. Most patients had a body mass index greater than 30 , had previous abdominal surgery, and had a small size hernia $(<4 \mathrm{~cm}$ width). The baseline demographics of the patient and characteristics of the hernias were similar between the two groups, with the exception of minor imbalances due to chance: more incisional hernias/previous abdominal surgeries in the RVHR group (88\% v 73\%) and more recurrent hernias in the LVHR group $(12 \% \mathrm{v}$ 25\%) (table 1).

The median follow-up for all patients was 6.4 (range 1-12) months. At one month after surgery, 123 (99\%) patients were evaluated in the clinic. One patient who underwent LVHR could not be seen or contacted by phone or mail and was therefore excluded from the analysis.

Primary fascial closure was achieved using suture only in all patients included in this study. None of the cases required abdominal wall component separation. Surgeon number 1 did 52 (42\%) of the procedures (29 RVHR and 23 LVHR), surgeon number 2 did 50 (40\%) of the procedures (24 RVHR and 26 LVHR), and surgeon number 3 did $22(18 \%)$ of the procedures (12 RVHR and 10 LVHR). However, this study was stratified by surgeon to ensure balance.

The median number of days in hospital at 90 days after surgery did not differ between groups (table 2). Details of patients who had postoperative length of stay of one day or longer and those who were readmitted are shown in supplementary table $\mathrm{C}$.

RVHR had significantly longer mean operating room times than did LVHR (141 v 77 min; $\mathrm{P}<0.001)$ (supplementary tables D1 and D2). Two RVHR cases were complicated with intra-operative inadvertent enterotomy. One patient with an incisional hernia after a Hartmann's procedure and subsequent colostomy takedown for perforated diverticulitis was found to have extensive intra-abdominal adhesions. Owing to concerns about possible enterotomy after extensive adhesiolysis, an exploratory laparotomy was performed. An enterotomy was found, and a small bowel resection and open ventral hernia repair with retromuscular mesh were performed. The second intra-operative enterotomy occurred in a patient with a recurrent ventral hernia after a previous repair with mesh. Several loops of small bowel were adhered to the previous mesh as well as incarcerated into the hernia. After reduction of the bowel, an enterotomy was identified and repaired through a robotic assisted enterorrhaphy. For this case, the hernia was repaired using sutures only without mesh. Although we did not initially consider inadvertent enterotomies in the secondary outcomes as none was anticipated in our trial, we decided to include them as one of the secondary results because of the clinical significance of these findings. Another patient in the LVHR group, with a large "Swiss-cheese" type incisional ventral hernia after a Hartmann's procedure and subsequent colostomy reversal for perforated diverticulitis, needed extensive lysis of adhesions. Owing to concerns about possible enterotomy, an exploratory laparotomy was performed. However, no injury was found and the hernia was repaired through an open approach with mesh (table 2).

We found no clear evidence of differences in conversions to open surgery, number of readmissions, emergency department visits, wound complications, or other complications. One surgical site infection occurred in a patient in the LVHR group who was treated with antibiotics and one reoperation that consisted of an incision and drainage of an abdominal wall seroma due to concern about surgical site infection. The fluid was clear, and the cultures were negative. At 90 days after surgery, the care of patients undergoing RVHR was significantly more expensive than for LVHR (\$15865 (£12 746; €14125) v \$12955; cost ratio 1.21, 95\% confidence interval 1.07 to 1.38 ; adjusted absolute cost difference $\$ 2767, \$ 910$ to $\$ 4626 ; \mathrm{P}=0.004$ ) (table 2 and supplementary tables E1 and E2).

At one month follow-up, the median abdominal wall quality of life scores of patients who underwent RVHR improved by only 3 points, whereas those in the LVHR group had a clinically important increase of 15 


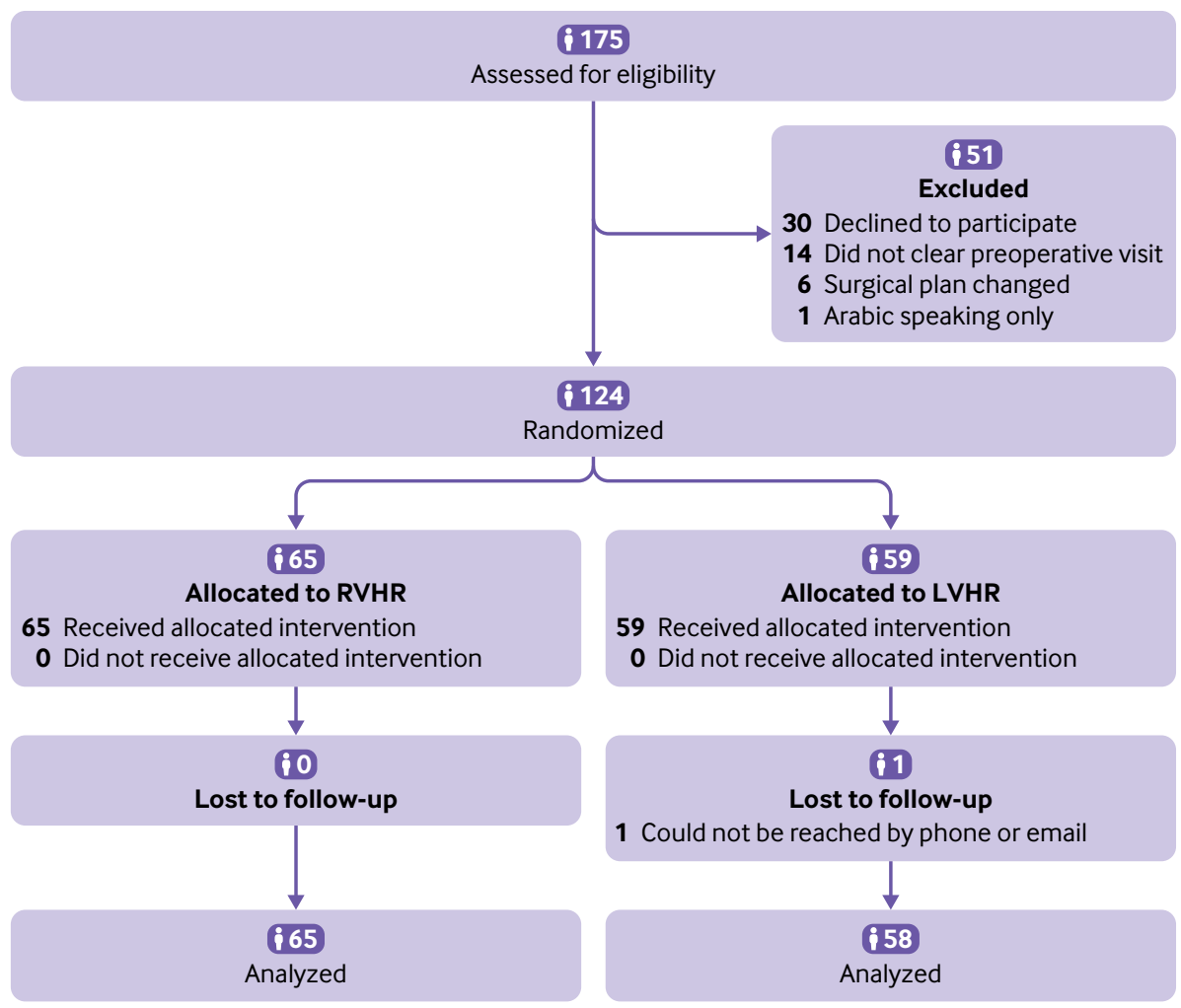

Fig 1 | CONSORT flow diagram. LVHR=laparoscopic ventral hernia repair; RVHR=robotic ventral hernia repair

points in their median scores (table 3; supplementary tables $\mathrm{F}$ and $\mathrm{G}$ ). Although confidence intervals are wide, a higher percentage of patients in the RVHR arm had a major worsening in abdominal wall quality of life $(28 \% \vee 14 \%$; relative risk 2.07, 0.98 to $4.41 ; \mathrm{P}=0.058$ ), whereas a higher percentage of patients in the LVHR group had a major improvement in abdominal wall quality of life (44\% v 53\%; relative risk $1.20,0.83$ to $1.74 ; \mathrm{P}=0.33$ ). We found no clear evidence of a difference in change of pain scores between groups at one month postoperative follow-up.

\section{Discussion}

This is the first multicenter randomized controlled trial comparing both clinical and patient centered outcomes between RVHR and LVHR. No evidence of a difference in length of stay was found between the groups. However, RVHR needed nearly twice as much operative time and increased costs to the healthcare system (even without accounting for the elevated acquisition costs of the robotic platform and equipment estimated at $\$ 0.5-2.5 \mathrm{~m}$ per platform and maintenance costs estimated at \$80000-190000 per platform per year). ${ }^{30-32}$ In addition, we found no clinical, patient centered, or economic benefit to RVHR compared with LVHR. The results of this study are in line with most other comparative studies published from large datasets comparing RVHR and LVHR: no clinical benefit, with increased operative duration and healthcare costs. ${ }^{33-36}$
This study unexpectedly found more enterotomies with RVHR (3\% v 0\% with LVRH). This outcome was not pre-specified (it was unexpected), was not statistically significant, and could be due to chance or a true finding. Therefore, we believe that a larger study is needed to corroborate this finding. Given our study results and assuming that the true effect size is $50 \%$ lower (that is, $2 \% v 0.5 \%$ risk of enterotomy), 1720 patients would be needed for an appropriately powered study to detect a true difference. Considering that more than 400000 ventral hernia repairs are done each year in the US, along with more than 6000 patients reported in the literature as having had RVHR, and that our results show no clinical benefit, larger multicenter randomized controlled trials are justified to assess the safety, efficacy, and effectiveness of RVHR before its further adoption.

During robotic intraperitoneal onlay mesh placement repair, the mesh is commonly fixated using a running suture. Alternatively, for laparoscopic repair, four cardinal sutures are commonly used, along with tacks in a single or double crown. We chose the most common surgical techniques endorsed by major surgical societies and acknowledge that some of the findings (operating room duration, patient centered outcomes) observed in our trial may be related to different fixation techniques. Multiple small randomized controlled trials have assessed mesh fixation in laparoscopic repair, resulting in systematic reviews showing conflicting results. When only studies at low risk of bias (that is, well performed randomized 


\begin{tabular}{|c|c|c|c|}
\hline Characteristics & Total $(n=124)$ & RVHR $(n=65)$ & $\operatorname{LVHR}(n=59)$ \\
\hline Mean (SD) age, years & $49.1(13.1)$ & $50.1(13.3)$ & $48.0(12.9)$ \\
\hline Female sex & $85(69)$ & $48(74)$ & $37(63)$ \\
\hline \multicolumn{4}{|l|}{ Race/ethnicity: } \\
\hline Hispanic & $95(77)$ & $50(77)$ & $45(76)$ \\
\hline African-American & $15(12)$ & $6(9)$ & $9(15)$ \\
\hline White & $10(8)$ & $7(11)$ & $3(5)$ \\
\hline Others & $4(3)$ & $2(3)$ & $2(3)$ \\
\hline Mean (SD) body mass index & $32.1(5.0)$ & $32.4(4.6)$ & $31.8(5.4)$ \\
\hline Obesity ${ }^{\star}$ & $86(69)$ & $45(69)$ & $41(69)$ \\
\hline Recent smokert & $2(2)$ & $2(3)$ & $0(0)$ \\
\hline Diabetes mellitus & $27(22)$ & $15(23)$ & $12(20)$ \\
\hline \multicolumn{4}{|l|}{ ASA class: } \\
\hline $1-2$ & $84(68)$ & $42(65)$ & $42(71)$ \\
\hline $3-4$ & $40(32)$ & $23(35)$ & $17(29)$ \\
\hline Previous abdominal surgery & $100(81)$ & $57(88)$ & $43(73)$ \\
\hline \multicolumn{4}{|l|}{ Hernia type: } \\
\hline Primary & $24(19)$ & $8(12)$ & $16(27)$ \\
\hline Incisional & $100(81)$ & $57(88)$ & $43(73)$ \\
\hline Recurrent & $23(19)$ & $8(12)$ & $15(25)$ \\
\hline Median (IQR) hernia width, cm & $3.0(1.5-5.0)$ & $3.0(2.0-5.0)$ & $3.0(1.0-4.5)$ \\
\hline \multicolumn{4}{|l|}{ Hernia width: } \\
\hline Small $(<4 \mathrm{~cm})$ & $88(71)$ & $44(68)$ & $44(75)$ \\
\hline Medium $(4-10 \mathrm{~cm})$ & $29(23)$ & $18(28)$ & $11(19)$ \\
\hline Large $(>10 \mathrm{~cm})$ & $7(6)$ & $3(5)$ & $4(7)$ \\
\hline
\end{tabular}

controlled trials) have been evaluated, no differences among the different mesh fixation methods has been clearly shown, except that fibrin glue fixation alone may increase hernia recurrence rates. ${ }^{37-41}$ Proponents of RVHR quote improved patient centered outcomes through a decrease in pain secondary to suturing rather than tacking of the mesh. ${ }^{42}$ However, our study did not support these results. At one month after surgery, LVHR was associated with a statistically nonsignificant improvement in abdominal wall quality of life compared with RVHR. We found no difference in pain scores. These findings may change over time, so

\begin{tabular}{|c|c|c|c|c|}
\hline Outcome & RVHR $(n=65)$ & $\operatorname{LVHR}(n=58)$ & $P$ value & Relative rate $(95 \% \mathrm{Cl})^{*}$ \\
\hline Median days in hospital at 90 days & 0 & 0 & 0.82 & $0.90(0.37$ to 2.19$)$ \\
\hline Postoperative length of stay & 0 & 0 & & \\
\hline Readmissions length of stay & 0 & 0 & & \\
\hline Days in hospital at 90 days (categories): & & & 0.28 & - \\
\hline 0 days & $50(77)$ & $49(84)$ & & \\
\hline 1 day & $9(14)$ & $4(7)$ & & \\
\hline 2 days & $4(6)$ & $1(2)$ & & \\
\hline 13 days & $2(3)$ & $4(7)$ & & \\
\hline Mean (SD) operating room duration, min & $141(56)$ & $77(37)$ & $<0.001$ & $62.89+(45.75$ to 80.01$)$ \\
\hline Wound class 2-4 & $2(3)$ & $0(0)$ & 0.50 & $\ddagger$ \\
\hline Conversion to open repair & $1(2)$ & $1(2)$ & 0.84 & $0.76(0.05$ to 11.47$)$ \\
\hline Patients readmitted & $1(2)$ & $3(5)$ & 0.25 & $0.27(0.03$ to 2.43$)$ \\
\hline Patients with emergency room visits & $7(11)$ & $5(9)$ & 0.66 & $1.28(0.43$ to 3.75$)$ \\
\hline Wound complication: & $13(20)$ & $11(19)$ & 0.95 & 1.02 (0.51 to 2.08$)$ \\
\hline Surgical site infection & $0(0)$ & $1(2)$ & 1.00 & \\
\hline Seroma & $13(20)$ & $8(14)$ & 0.40 & \\
\hline Hematoma & $0(0)$ & $2(3)$ & 1.00 & \\
\hline Recurrence & $0(0)$ & $0(0)$ & 1.00 & $\ddagger$ \\
\hline Reoperation & $0(0)$ & $1(2)$ & 1.00 & $\ddagger$ \\
\hline Clavien-Dindo complications: & $14(22)$ & $11(19)$ & 0.80 & $1.10(0.54$ to 2.24$)$ \\
\hline $1-2$ & $14(22)$ & $10(17)$ & & \\
\hline $3-5$ & $0(0)$ & $1(2) \S$ & & \\
\hline Mean (SD) costs, US\$ & $15865(4879)$ & $12955(5636)$ & 0.004 & $1.219(1.07$ to 1.38$)$ \\
\hline \multicolumn{5}{|c|}{$\begin{array}{l}\text { LVHR=laparoscopic ventral hernia repair; RVHR=robotic ventral hernia repair. } \\
\text { *LVHR is control for relative rate calculation. } \\
\text { tMean differences are reported instead of relative rate as variable is continuous and was analyzed with generalized linear model. } \\
\text { †Relative rate could not be calculated as no observations in one arm. } \\
\text { SRepresents incision and drainage of abdominal wall seroma. }\end{array}$} \\
\hline
\end{tabular}


we plan to publish long term follow-up results in the future.

New innovations can be disruptive in both a positive and a negative fashion. For example, an era of smaller incisions, fewer incisional hernias, improved quality of life for patients, shorter length of stay in hospital, improved recovery, and improved return to work came with the introduction of laparoscopy. However, during its early adoption, laparoscopy also resulted in more major complications such as enterotomies, bile duct injuries, and major vascular injuries. Although robotics will probably be the future of surgery in the long term, the current technology does not seem to be superior to a skilled laparoscopic surgeon for ventral hernia repair and may in fact be inferior. With time, as the technology improves, robotics may surpass skilled surgeons. However, for now, RVHR does not seem to be justified, and its use should be limited to centers conducting randomized controlled trials.

\section{Strengths and limitations of study}

This study has several strengths. The main strength is a study design with a low risk of bias, as patients were randomly assigned to their treatment just before the operation, and both patients and postoperative outcome assessors were blinded to the allocation. To increase the generalizability of our results to a diversity of patients, we designed our study with wide inclusion criteria. Another key strength of our study is the completion of a ramp-up period before the start of the trial to ensure optimal workflow and limit the learning curve effect of the standardized technique used for this study. Lastly, a high rate of follow-up was achieved, and although this is common for short term outcomes, we are doing one year, three year, and five year followups to assess long term outcomes such as recurrence of hernia and quality of life and pain scores, and we plan to publish these in a later manuscript.
We acknowledge some limitations to this study. The study was carried out by experts in minimally invasive surgery, so the generalizability of these results to surgeons with lower volumes of such surgery may be limited. However, given the relation between case volume and outcomes, imagining that outcomes may be worse among surgeons with lower volumes is reasonable. Most hernia defects included in our study were small to medium in size. Some surgeons argue that the advantage of robotic ventral hernia repair is seen in patients with larger hernias that may need abdominal component separation techniques (for example, transversus abdominis release) to achieve primary fascial closure. However, no high quality studies have been done in this population to validate this statement. Furthermore, many more RVHRs are performed on small and medium size hernias. Thus, we chose to investigate this patient population first as results would have the greatest applicability and affect a larger number of patients. In addition, we included both primary ventral and ventral incisional hernias, which may have different outcomes. However, on subgroup analysis, this variable did not affect the findings. Moreover, during this trial we placed the mesh in the intraperitoneal location, which is the most common approach for small, uncomplicated ventral hernias. Mesh placement in the retrorectus space has been found to be the location associated with fewest hernia recurrences and surgical site infections during open ventral hernia repair ${ }^{43}$; however, randomized trials designed to compare alternate mesh positions during robotic or laparoscopic repair are lacking. Proponents argue that another advantage of robotic repair is to allow easier placement of mesh in spaces other than intraperitoneally (for example, preperitoneal, retrorectus), and interest in techniques such as extended view total extraperitoneal repair and transabdominal preperitoneal repair has been increasing.

\begin{tabular}{|c|c|c|c|c|}
\hline & RVHR $(n=64)$ & $\operatorname{LVHR}(\mathrm{N}=5)$ & Difference in median $(95 \% \mathrm{Cl})$ & Pvalue \\
\hline \multicolumn{5}{|c|}{ Median (IQR) AW-QOL scores (mAAS) } \\
\hline Baseline & $48(27-71)$ & $45(12-63)$ & - & - \\
\hline Follow-up & $52(37-68)$ & $65(36-86)$ & $8.25(-1.75$ to 20.00$)$ & 0.13 \\
\hline Change & $3(-16-24)$ & $15(-4-38)$ & $11.00(-0.33$ to 21.08$)$ & 0.06 \\
\hline ANCOVA & & & $-9.15(-18.57$ to 0.26$)$ & 0.06 \\
\hline \multicolumn{5}{|c|}{ No (\%) in AW-QOL change category } \\
\hline Worsened: & $22(34)$ & $12(20)$ & $1.69^{\star}(0.92$ to 3.10$)$ & \multirow[t]{7}{*}{0.22} \\
\hline Major worsening («-14) & $18(28)$ & $8(14)$ & & \\
\hline Minor worsening (८-7) & $4(6)$ & $4(7)$ & & \\
\hline Improved: & $30(47)$ & $34(58)$ & $0.81^{*}(0.58$ to 1.14$)$ & \\
\hline Major improvement (>14) & $28(44)$ & $31(53)$ & & \\
\hline Minor improvement (>7) & $2(3)$ & $3(5)$ & & \\
\hline No change & $12(19)$ & $13(22)$ & $0.85^{\star}(0.42$ to 1.71$)$ & \\
\hline \multicolumn{5}{|c|}{ Median (IQR) pain scores (VAS) } \\
\hline Baseline & $3.0(0-6)$ & $3.5(0-6)$ & - & - \\
\hline Follow-up & $4.0(2-6)$ & $4.0(0-6)$ & $0.00(-0.00$ to 1.00$) \dagger$ & 0.43 \\
\hline Change & $0(-1-3)$ & $0(-2-2)$ & $0.00(-0.00$ to 1.00$) \dagger$ & 0.59 \\
\hline ANCOVA & & & $0.42(-0.58$ to 1.42$)$ & 0.40 \\
\hline \multicolumn{5}{|c|}{$\begin{array}{l}\text { ANCOVA=analysis of covariance; AW-QOL=abdominal wall quality of life; IQR=interquartile range; } m A A S=\text { modified Activity Assessment Scale (range fror } \\
1=\text { poor to } 100=\text { perfect; minimal clinically important difference }(M C I D)=7) ; \mathrm{LVHR}=\text { laparoscopic ventral hernia repair; RVHR=robotic ventral hernia repair } \\
\text { VAS=visual analog scale (range from } 0=\text { no pain to } 10=\text { =worst possible pain; } M C I D=1) \text {. } \\
\text { * Relative risk is reported instead of difference in median as variable is categorical. } \\
\text { tConfidence interval could not be accurately calculated owing to extremely small differences in medians. }\end{array}$} \\
\hline
\end{tabular}


However, only a limited number of surgeons are using these approaches, and only studies at high risk of bias (for example, retrospective reviews and case series) have been published on these topics. ${ }^{44-46}$ We advocate for future randomized trials to assess RVHR of large hernias and approaches other than the intraperitoneal onlay mesh placement technique before widespread dissemination and adoption. Finally, to increase the precision of our estimates, length of stay could have been measured in hours instead of days. However, what the minimum clinically important difference is for hours rather than days in the context of ventral hernia repair is unclear.

\section{Conclusion}

This is the first randomized controlled trial comparing robotic versus laparoscopic ventral hernia repair. Among patients undergoing minimally invasive ventral hernia repair, we found no evidence of a difference in 90 day postoperative hospital days between robotic and laparoscopic repair. Furthermore, robotic surgery nearly doubled operating room time and significantly increased the cost of healthcare. Larger multicenter trials are needed before widespread adoption of robotic ventral hernia repair can be recommended.

\section{AUTHOR AFFILIATIONS}

Department of Surgery, Lyndon B. Johnson General Hospital, McGovern Medical School at UTHealth, Houston, TX 77026, USA

${ }^{2}$ Center for Surgical Trials and Evidence-Based Practice, McGovern Medical School at UTHealth, Houston, TX, USA

${ }^{3}$ Department of Surgery, Memorial Hermann Texas Medical Center, McGovern Medical School at UTHealth, Houston, TX, USA

${ }^{4}$ Department of Pediatrics, McGovern Medical School at UTHealth, Houston, TX, USA

${ }^{5}$ Center for Clinical Research and Evidence Based Medicine, McGovern Medical School at UTHealth, Houston, TX, USA

${ }^{6}$ Michael E. DeBakey Department of Surgery, Baylor College of Medicine, Houston, TX, USA

Contributors: OAO, KB, SKS, TDW, TCK, LSK, and MKL were responsible for study conception and design. OAO, KB, SKS, TDW, SW, MML, and MKL acquired the data. OAO, KB, SKS, TDW, SW, CP, EBA, MML, TCK, LSK, and MKL analyzed and interpreted the data. OAO, KB, SKS, TDW, MML, LSK, and MKL drafted the manuscript. OAO, KB, SKS, TDW, CP, EBA, MML, TCK, LSK, and MKL critically revised the manuscript. The corresponding author attests that all listed authors meet authorship criteria and that no others meeting the criteria have been omitted. OAO and MKL are the guarantors.

Funding: This study was supported by an investigator initiated grant from Intuitive Surgical. This funding source had no role in the design, data collection, analysis, interpretation, writing, or decision to submit the results of this study. All authors had full access to the data and are responsible for the decision to submit for publication.

Competing interests: All authors have completed the ICMJE uniform disclosure form at www.icmje.org/coi_disclosure.pdf and declare: support from Intuitive Surgical for the submitted work; SKS receives consulting fees from C-SATS, educational honorariums from Gore, and past research grant support from Neosurgical and Medigus; TDW receives consulting fees from CONMED, EndoEvolution, Davol, and Livsmed and is on the advisory board of ColubrisMX; no other relationships or activities that could appear to have influenced the submitted work.

Ethical approval: This study was approved by the institutional review board of the University of Texas Health Science Center at Houston under the protocol ID number HSC-MS-18-0137. Participants signed a surgical informed consent and a separate research consent before enrollment.

Data sharing: De-identified individual patient information, data dictionary defining each field in the dataset, study protocol, and statistical analysis plan and code will be available after publication through individual request directed to the corresponding author, to researchers whose proposed use of the data has been approved.

The lead author affirms that the manuscript is an honest, accurate, and transparent account of the study being reported; that no important aspects of the study have been omitted; and that any discrepancies from the study as planned (and, if relevant, registered) have been explained.

Dissemination to participants and related patient and public communities: Participating institutions and surgical departments were informed of study results through grand round conferences. Results of the study will be communicated by the principal investigator to those patients enrolled in the study who express interest in the results in future clinic visits. The results were presented to the surgical community at the American College of Surgeons Clinical Congress in San Francisco, California, in October 2019 (https://www.facs.org/ clincon2019/resources/highlights/mon/lbct02?fbclid=IwAR2FhzR8 Tgy1wIJxw38GUwN-rKjxqu42qaC_iWpD8UucnJqDX_ZplUAXIpo) and reported on the front page of General Surgery News (https://www. generalsurgerynews.com/In-the-News/Article/12-19/RandomizedTrial-Pits-Laparoscopy-Against-Robot-for-Ventral-Hernia/56790). Additionally, the information was presented at the Intuitive Surgical (funding source) Annual Research Symposium held in Sunnyvale, California, in January 2020.

This is an Open Access article distributed in accordance with the Creative Commons Attribution Non Commercial (CC BY-NC 4.0) license, which permits others to distribute, remix, adapt, build upon this work non-commercially, and license their derivative works on different terms, provided the original work is properly cited and the use is noncommercial. See: http://creativecommons.org/licenses/by-nc/4.0/.

1 Intuitive Surgical, Inc. Annual Report 2018. 2018. http://www. annualreports.com/HostedData/AnnualReportArchive/i/NASDAO ISRG_2018.pdf.

2 Patel SV, Yu D, Elsolh B, Goldacre BM, Nash GM. Assessment of Conflicts of Interest in Robotic Surgical Studies: Validating Author's Declarations With the Open Payments Database. Ann Surg 2018;268:86-92. doi:10.1097/SLA.0000000000002420

3 Prabhu AS, Dickens EO, Copper CM, et al. Laparoscopic vs Robotic Intraperitoneal Mesh Repair for Incisional Hernia: An Americas Hernia Society Quality Collaborative Analysis. J Am Coll Surg 2017;22 25:28593. doi:10.1016/j.jamcollsurg.2017.04.011

4 Shah SK, Liang MK. Laparoscopic ventral hernia repair. 2018. Available from: https://www.uptodate.com/contents/laparoscopicventral-hernia-repair.

5 Alawadi ZM, Leal IM, Flores JR, et al. Underserved Patients Seeking Care for Ventral Hernias at a Safety Net Hospital: Impact on Quality o Life and Expectations of Treatment. J Am Coll Surg 2017;224:26-34. e2. doi:10.1016/j.jamcollsurg.2016.09.017

6 Henchcliffe BE, Holihan IL, Flores-Gonzalez JR, et al. Barriers to Participation in Preoperative Risk-Reduction Programs Prior to Ventral Hernia Repair: An Assessment of Underserved Patients at a Safety-Net Hospital. JAMA Surg 2016;151:488-90. doi:10.1001/ jamasurg.2015.4452

7 Krpata DM, Schmotzer BJ, Flocke S, et al. Design and initial implementation of HerQLes: a hernia-related quality-of-life survey to assess abdominal wall function. J Am Coll Surg 2012;215:635-42. doi:10.1016/j.jamcollsurg.2012.06.412

8 McCarthy MJr, Jonasson $\mathrm{O}$, Chang $\mathrm{CH}$, et al. Assessment of patient functional status after surgery. J Am Coll Surg 2005;201:171-8. doi:10.1016/j.jamcollsurg.2005.03.035

9 Cherla DV, Moses ML, Viso CP, et al. Impact of Abdominal Wall Hernias and Repair on Patient Quality of Life. World J Surg 2018;42:19-25. doi:10.1007/s00268-017-4173-6

10 Poulose BK, Roll S, Murphy JW, et al. Design and implementation of the Americas Hernia Society Quality Collaborative (AHSOC): improving value in hernia care. Hernia 2016;20:177-89. doi:10.1007/s10029-016-1477-7

11 Myles PS, Myles DB, Galagher W, et al. Measuring acute postoperative pain using the visual analog scale: the minimal clinically important difference and patient acceptable symptom state. $\mathrm{Br}$ J Anaesth 2017;118:424-9. doi:10.1093/bja/aew466

12 Liang MK, Holihan IL, Itani K, et al. Ventral Hernia Management: Expert Consensus Guided by Systematic Review. Ann Surg 2017;265:80-9. doi:10.1097/SLA.0000000000001701

13 Pernar LIM, Robertson FC, Tavakkoli A, Sheu EG, Brooks DC, Smink DS. An appraisal of the learning curve in robotic general surgery. Surg Endosc 2017;31:4583-96. doi:10.1007/s00464-017-5520-2

14 Herron DM, Marohn M, SAGES-MIRA Robotic Surgery Consensus Group. A consensus document on robotic surgery. Surg Endosc 2008;22:313-25, discussion 311-2. doi:10.1007/s00464007-9727-5 
15 Walker PA, May AC, Mo J, et al. Multicenter review of robotic versus laparoscopic ventral hernia repair: is there a role for robotics? Surg Endosc 2018;32:1901-5. doi:10.1007/s00464-017-5882-5

16 Olavarria OA, Bernardi K, Wilson TD, et al. Safety and Feasibility of Robotic Surgery in a County Hospital. Gastroenterology 2019;156:1480. doi:10.1016/S00165085(19)40773-7

17 Society of American Gastrointestinal and Endoscopic Surgeons (SAGES). Laparoscopic ventral hernia repair patient information from SAGES. 2015. https://www.sages.org/publications/patientinformation/patient-information-for-laparoscopic-ventral-herniarepair-from-sages/.

18 Patel A, Oleynikov D, eds. The SAGES Manual of Robotic Surgery. Springer, 2018. doi:10.1007/978-3-319-51362-1

19 Bernardi K, Olavarria OA, Holihan JL, et al. Primary Fascial Closure During Laparoscopic Ventral Hernia Repair Improves Patient Quality of Life: A Multicenter, Blinded Randomized Controlled Trial. Ann Surg 2020;271:434-9. doi:10.1097/SLA.0000000000003505

20 Ahonen-Siirtola M, Nevala T, Vironen J, et al. Laparoscopic versus hybrid approach for treatment of incisional ventral hernia: a prospective randomized multicenter study of 1-month follow-up results. Hernia 2018;22:1015-22. doi:10.1007/s10029-018-1784-2

21 Ahonen-Siirtola M, Nevala T, Vironen J, et al. Laparoscopic versus hybrid approach for treatment of incisional ventral hernia: a prospective randomised multicentre study, 1-year results. Surg Endosc 2020:34:88-95 doi:10.1007/s00464-019-06735-9

22 Mangram AJ, Horan TC, Pearson ML, Silver LC, Jarvis WR, Centers for Disease Control and Prevention (CDC) Hospital Infection Control Practices Advisory Committee. Guideline for Prevention of Surgical Site Infection, 1999. Am J Infect Control 1999;27:97-132, quiz 133 4, discussion 96. doi:10.1016/S0196-6553(99)70088-X

23 Breuing K, Butler CE, Ferzoco S, et al, Ventral Hernia Working Group. Incisional ventral hernias: review of the literature and recommendations regarding the grading and technique of repair. Surgery 2010;148:544-58. doi:10.1016/j.surg.2010.01.008

24 American College of Surgeons National Surgical Quality Improvement Program. User Guide for the 2017 ACS NSQIP Participant Use Data File (PUF). 2018. https://www.facs.org/ /media/files/quality\%20 programs/nsqip/nsqip puf userguide 2017.ashx.

25 Diehr P, Yanez D, Ash A, Hornbrook M, Lin DY. Methods for analyzing health care utilization and costs. Annu Rev Public Health 1999;20:125-44. doi:10.1146/annurev.publhealth.20.1.125

26 Kahan BC. Accounting for centre-effects in multicentre trials with a binary outcome - when, why, and how?BMC Med Res Methodol 2014;14:20. doi:10.1186/1471-2288-14-20

27 Kahan BC, Morris TP. Improper analysis of trials randomised using stratified blocks or minimisation. Stat Med 2012;31:328-40. doi:10.1002/sim.4431

28 Vickers AJ, Altman DG. Statistics notes: Analysing controlled trials with baseline and follow up measurements. BMJ 2001:323:1123-4. doi:10.1136/bmj.323.7321.1123

29 The R Foundation. The R Project for Statistical Computing. 2019. https://www.R-project.org/.

30 Childers CP, Maggard-Gibbons M. Estimation of the Acquisition and Operating Costs for Robotic Surgery. JAMA 2018;320:835-6. doi:10.1001/jama.2018.9219

31 Barbash GI, Glied SA. New technology and health care costs--the case of robot-assisted surgery. N Engl J Med 2010;363:701-4. doi:10.1056/NEJMp1006602
32 Intuitive Surgical. Intuitive Surgical investor presentation, Q2 2020. https://isrg.gcs-web.com/static-files/7b0470fb-cfd2-456a-b6eb24af76d68f6d.

33 Zayan NE, Meara MP, Schwartz JS, Narula VK. A direct comparison of robotic and laparoscopic hernia repair: patient-reported outcomes and cost analysis. Hernia 2019;23:1115-21. doi:10.1007/s10029019-01943-7

34 Henriksen NA, Jensen KK, Muysoms F. Robot-assisted abdominal wall surgery: a systematic review of the literature and meta-analysis. Hernia 2019;23:17-27. doi:10.1007/s10029-018-1872-3

35 Khorgami Z, Li WT, Jackson TN, Howard CA, Sclabas GM. The cost of robotics: an analysis of the added costs of robotic-assisted versus laparoscopic surgery using the National Inpatient Sample. Surg Endosc 2019;33:2217-21. doi:10.1007/s00464-018-6507-3

36 Altieri MS, Yang J, Xu J, Talamini M, Pryor A, Telem DA. Outcomes after Robotic Ventral Hernia Repair: A Study of 21,565 Patients in the State of New York. Am Surg 2018;84:902-8. doi:10.1177/000313481808400639

37 Ahmed MA, Tawfic QA, Schlachta CM, Alkhamesi NA. Pain and Surgical Outcomes Reporting After Laparoscopic Ventral Hernia Repair in Relation to Mesh Fixation Technique: A Systematic Review and Meta-Analysis of Randomized Clinical Trials. J Laparoendosc Adv Surg Tech A 2018;28:1298-315. doi:10.1089/lap.2017.0609

38 Khan RMA, Bughio M, Ali B, Hajibandeh S, Hajibandeh S. Absorbable versus non-absorbable tacks for mesh fixation in laparoscopic ventral hernia repair: A systematic review and meta-analysis. Int J Surg 2018;53:184-92. doi:10.1016/j.ijsu.2018.03.042

39 Baker JJ, Öberg S, Andresen K, Klausen TW, Rosenberg J. Systematic review and network meta-analysis of methods of mesh fixation during laparoscopic ventral hernia repair. Br J Surg 2018;105:37-47. doi:10.1002/bjs.10720

40 Reynvoet E, Deschepper E, Rogiers X, Troisi R, Berrevoet F. Laparoscopic ventral hernia repair: is there an optimal mesh fixation technique? A systematic review. Langenbecks Arch Surg 2014;399:55-63. doi:10.1007/s00423-013-1126-x

41 Brill JB, Turner PL. Long-term outcomes with transfascial sutures versus tacks in laparoscopic ventral hernia repair: a review. Am Surg 2011;77:458-65. doi:10.1177/000313481107700423

42 Bansal VK, Misra MC, Kumar S, et al. A prospective randomized study comparing suture mesh fixation versus tacker mesh fixation for laparoscopic repair of incisional and ventral hernias. Surg Endosc 2011;25:1431-8. doi:10.1007/s00464-010-1410-6

43 Holihan JL, Hannon C, Goodenough C, et al. Ventral Hernia Repair: A Meta-Analysis of Randomized Controlled Trials. Surg Infect (Larchmt) 2017;18:647-58. doi:10.1089/sur.2017.029

44 Orthopoulos G, Kudsi OY. Feasibility of Robotic-Assisted Transabdominal Preperitoneal Ventral Hernia Repair. J Laparoendosc Adv Surg Tech A 2018;28:434-8. doi:10.1089/lap.2017.0595

45 Belyansky I, Reza Zahiri H, Sanford Z, Weltz AS, Park A. Early operative outcomes of endoscopic (eTEP access) robotic-assisted retromuscular abdominal wall hernia repair. Hernia 2018;22:837 47. doi:10.1007/s10029-018-1795-z

46 Lu R, Addo A, Ewart Z, et al. Comparative review of outcomes: laparoscopic and robotic enhanced-view totally extraperitoneal (eTEP) access retrorectus repairs. Surg Endosc 2019. doi:10.1007/ s00464-019-07132-y

Web appendix: Supplementary tables 